\title{
A 3D 3-Subiteration Thinning Algorithm for Medial Surfaces
}

\author{
Kálmán Palágyi \\ Department of Applied Informatics, University of Szeged, \\ H-6701 Szeged P.O.Box 652, Hungary \\ palagyi@inf.u-szeged.hu
}

\begin{abstract}
Thinning on a binary picture is an iterative layer by layer erosion to extract a reasonable approximation to its skeleton. This paper presents an efficient 3D parallel thinning algorithm which produces medial surfaces. Three-subiteration directional strategy is proposed: each iteration step is composed of three parallel subiterations according to the three deletion directions. The algorithm makes easy implementation possible, since deletable points are given by matching templates containing twentyeight elements. The topological correctness of the algorithm for $(26,6)$ binary pictures is proved.
\end{abstract}

\section{Introduction}

Skeletonization provides shape features that are extracted from binary image data. A very illustrative definition of the skeleton is given using the prairie-fire analogy: the object boundary is set on fire and the skeleton is formed by the loci where the fire fronts meet and quench each others [3]. In discrete spaces, the thinning process is a frequently used method for producing an approximation to the skeleton in a topology-preserving way [6]. It based on digital simulation of the fire front propagation: border points of a binary object that satisfy certain topological and geometric constraints [21] are deleted in iteration steps. The entire process is repeated until only the "skeleton" is left. Therefore, a thinning algorithm can be regarded as a reduction operation that changes some 1's (object elements or black points) to 0's (white points) but does not alter 0's.

A reduction operation does not preserve topology if

- any object in the input picture is split (into two or more) or completely deleted,

- any cavity in the input picture is merged with the background or another cavity, or

- a cavity is created where there was none in the input picture.

There is an additional concept called hole in 3D pictures. A hole (that doughnuts have) is formed by 0 's, but it is not a cavity [6]. Topology preservation implies that eliminating or creating holes is not allowed. 
A simple point is an object point whose deletion does not alter the topology of the picture [13]. Sequential thinning algorithms delete simple points which are not end points, since preserving end-points provides important information relative to the shape of the objects. Curve thinning (i.e., a thinning process for extracting medial line) preserves line-end points while surface thinning (i.e., a thinning process for extracting medial surface) does not delete surface-end points.
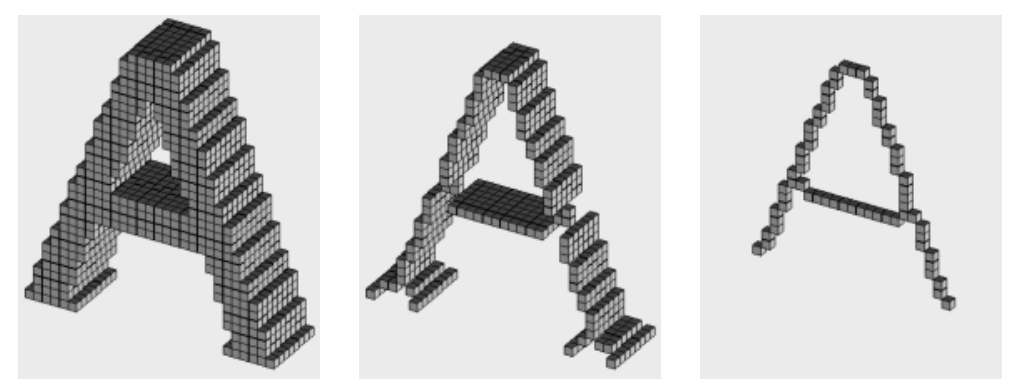

Fig. 1. A 3D synthetic picture containing a character "A" (left), result of a surface thinning process (centre), and result of a curve thinning process (right). (Cubes represent black points.)

Parallel thinning algorithms delete a set of simple points. A possible approach to preserve topology is to use directional strategy; each iteration step is composed of a number of parallel subiterations where only border points of certain kind can be deleted in each subiteration. There are six kinds of border points in 3D pictures on cubic grid, therefore, 6-subiteration directional thinning algorithms were generally proposed.

In this paper, a 3-subiteration directional algorithm is proposed for surface thinning. Some experiments are made on synthetic objects and the topology preservation for $(26,6)$ binary pictures [6] is proved. Our approach demonstrates a possible way for constructing non-conventional directional thinning algorithms.

\section{Basic Notions and Results}

Let $p$ be a point in the $3 \mathrm{D}$ digital space $Z^{3}$. Let us denote $N_{j}(p)$ (for $j=$ $6,18,26)$ the set of points $j$-adjacent to point $p$ (see Fig. 2). The sequence of distinct points $\left\langle x_{0}, x_{1}, \ldots, x_{n}\right\rangle$ is a $j$-path of length $n \geq 0$ from point $x_{0}$ to point $x_{n}$ in a non-empty set of points $X$ if each point of the sequence is in $X$ and $x_{i}$ is $j$-adjacent to $x_{i-1}$ for each $1 \leq i \leq n$. (Note that a single point is a $j$-path of length 0 .) Two points are $j$-connected in the set $X$ if there is a $j$-path in $X$ between them. A set of points $X$ is $j$-connected in the set of points $Y \supseteq X$ if any two points in $X$ are $j$-connected in $Y$. 
The $3 D$ binary $(m, n)$ digital picture $\mathcal{P}$ is a quadruple $\mathcal{P}=\left(\mathbb{Z}^{3}, m, n, B\right)[6]$. Each element of $\mathbb{Z}^{3}$ is called a point of $\mathcal{P}$. Each point in $B \subseteq \mathbb{Z}^{3}$ is called a black point and value 1 is assigned to it. Each point in $Z^{3} \backslash B$ is called a white point and value 0 is assigned to it. Adjacency $m$ belongs to the black points and adjacency $n$ belongs to the white points. A black component (or object) is a maximal $m$-connected set of points in $B$. A white component is a maximal $n-$ connected set of points in $B \subseteq Z^{3}$.

We are dealing with $(26,6)$ pictures. It is assumed that any picture contains finitely many black points.

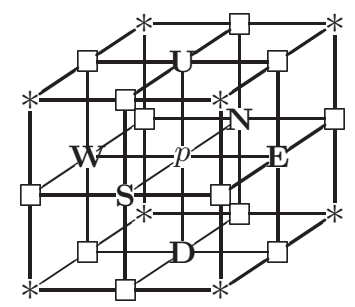

Fig. 2. The frequently used adjacencies in $Z^{3}$. The set $N_{6}(p)$ contains the central point $p$ and the 6 points marked $\mathbf{U}, \mathbf{D}, \mathbf{N}, \mathbf{E}, \mathbf{S}$, and $\mathbf{W}$. The set $N_{18}(p)$ contains the set $N_{6}(p)$ and the 12 points marked " $\square$ ". The set $N_{26}(p)$ contains the set $N_{18}(p)$ and the 8 points marked "*"

A black point is called border point if it is 6-adjacent to at least one white point. (Note that this definition is correct only for the special cases $m=26$ and $m=18$.) A border point $p$ is called $\mathbf{U}$-border point if the point marked by $\mathbf{U}$ in Fig. 2 is white. We can define $\mathbf{N}_{-}, \mathbf{E}-, \mathbf{S}_{-}, \mathbf{W}-$, and $\mathbf{D}$-border points in the same way.

A black point is called simple point if its deletion does not alter the topology of the picture. We make use of the following result for $(26,6)$ pictures:

Theorem 1. [12,19] Black point $p$ is simple in picture $\left(\mathbb{Z}^{3}, 26,6, B\right)$ if and only if all of the following conditions hold:

1. the set $(B \backslash\{p\}) \cap N_{26}(p)$ contains exactly one 26-component; and

2. the set $\left(Z^{3} \backslash B\right) \cap N_{6}(p)$ is not empty and it is 6-connected in the set $\left(Z^{3} \backslash B\right) \cap N_{18}(p)$.

Theorem 1 shows that the simplicity in $(26,6)$ pictures is a local property; it can be decided using the $3 \times 3 \times 3$ neighborhood of a given point.

We need to consider what is meant by topology preservation when a number of black points are deleted simultaneously. Ma [8] and Kong [5] gave sufficient conditions for parallel reduction operations of $3 \mathrm{D}(26,6)$ pictures. We use the following more general sufficient conditions: 
Theorem 2. [17,18] Let $\mathcal{T}$ be a parallel reduction operation on $(26,6)$ pictures. Then $\mathcal{T}$ is topology preserving, if for all picture $\mathcal{P}=\left(Z^{3}, 26,6, B\right)$, all of the following conditions hold:

1. for all points $p \in B$ that are deleted by $\mathcal{T}$ and for all sets $Q \subseteq\left(N_{18}(p) \backslash\{p\}\right) \cap$ $B$ that are deleted by $\mathcal{T}, p$ is simple in the picture $\left(\mathbb{Z}^{3}, 26,6, B \backslash Q\right)$; and

2. no black component contained entirely in a unit lattice cube (i.e., a $2 \times 2 \times$ 2 configuration in $\mathbb{Z}^{3}$ ) can be deleted completely by $\mathcal{T}$.

We propose a surface thinning algorithm. The deletable points of the algorithm are border points of certain types and not surface end-points (i.e., which are not extremities of surfaces). The proposed algorithm uses the following characterization of the the surface end-points.

Definition 1. $[17,18]$ The set $N_{6}(p)$ is subdivided into three kinds of opposite pairs of points (U,D), (N,S), and (E,W) (see Fig. 2).

$A$ black point $p$ is a surface end-point in a picture if the set $N_{6}(p)$ contains at least one opposite pair of white points.

\section{Existing Parallel Thinning Algorithms}

Most of the existing thinning algorithms are parallel, since the fire front propagation is by nature parallel. Those algorithms delete a set of simple points simultaneously that can alter the topology. There are three major strategies to overcome this problem:

\section{- Fully parallel algorithms:}

Algorithms from this group do not divide an iteration step into subiterations. In order to preserve topology, the known three fully parallel $3 \mathrm{D}$ thinning algorithms investigate larger neighborhood than the $3 \times 3 \times 3$ one: Ma proposed an algorithm, in which the new value of a black point depends on 30 points (and a parallel rechecking pass is required) [9], the fully parallel algorithm of Ma and Sonka uses a special neighborhood containing 50 points [10], and Manzanera et al. developed an algorithm using a symmetric neighborhood consisting of 81 points [11].

- Directional (or border sequential) algorithms:

Iteration steps are divided into a number of successive subiterations, where only border points of certain kind can be deleted in parallel in each subiteration. Consequently, each subiteration uses different deletion rule. Since there are six kinds of major directions in $3 \mathrm{D}$ pictures, 6-subiteration directional thinning algorithms were generally proposed $[2,4,7,14,15,22]$. Note that Palágyi and Kuba developed 8-subiteration [17] and 12-subiteration [18] directional thinning algorithms, too. Each existing directional algorithm examines the $3 \times 3 \times 3$ neighborhood of each border point. 


\section{- Subfield sequential algorithms:}

The $3 \mathrm{D}$ digital space $\mathbb{Z}^{3}$ is subdivided into more disjoint subfields that are alternatively activated. At a given iteration step, only border points in the active subfield are designated to be deleted. Each subiteration is executed in parallel (i.e., all border points in the actual subfield satisfying the deletion condition are simultaneously deleted). Two subfield sequential 3D thinning algorithms working in cubic grid has been proposed so far [1,20]. Both algorithms investigate the $3 \times 3 \times 3$ neighborhood and use eight subfields, therefore, each iteration step contains eight successive subiterations. Note that Palágyi and Kuba proposed a hybrid thinning algorithm [16]. It uses both subfield sequential and directional approaches (with two subfields and eight deletion directions).

The algorithm proposed in this paper follows the directional strategy. It requires only three subiterations (corresponding to the three kinds of opposite pair of points) in each iteration step, but an additional point not in the $3 \times 3 \times$ 3 neighborhood is examined in each subiteration.

\section{The New Thinning Algorithm}

In this section, a new algorithm is presented for extracting medial surfaces from $3 \mathrm{D}(26,6)$ pictures.

Each conventional 6-subiteration directional thinning algorithm uses the six deletion directions that can delete certain $\mathbf{U}_{-}, \mathbf{D}-, \mathbf{N}-, \mathbf{E}-, \mathbf{S}_{-}$, and $\mathbf{W}$-border points, respectively $[2,4,7,14,15,22]$. In our 3-subiteration approach, two kinds of border points can be deleted in each subiteration. The three deletion directions correspond to the three kinds of opposite pairs of points, and are denoted by UD, NS, and EW.

Suppose that the $3 \mathrm{D}(26,6)$ picture to be thinned contains finitely many back points. Reduction operations associated with the three subiterations are called deletion_from_UD, deletion_from_NS, and deletion_from_EW. We are now ready to present the 3 -subiteration approach formally:

Input: picture $\mathcal{P}=\left(\mathbb{Z}^{3}, 26,6, B\right)$

Output: picture $\mathcal{P}^{\prime}=\left(\mathbb{Z}^{3}, 26,6, B^{\prime}\right)$

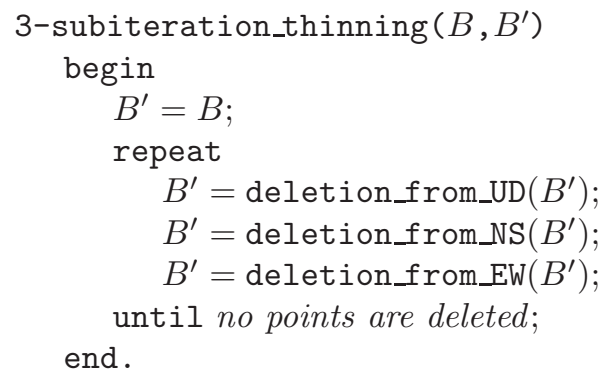


The new value of a black point depends on 28 points in each subiteration. The three special neighborhoods assigned to the different subiterations are presented in Fig. 3.
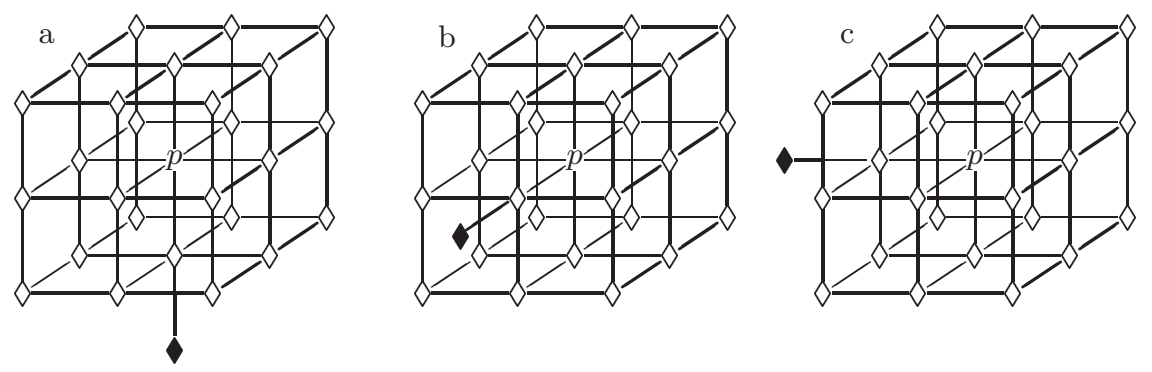

Fig. 3. The special local neighborhoods assigned to the deletion directions UD (a), NS (b), and EW (c), respectively. The new value of a black point $p$ depends on the $3 \times 3 \times 3$ neighborhood of $p$ (marked " $\diamond$ ") and an additional point (marked " $)$ ") that is not in $N_{26}(p)$

Deletable points in a subiteration are given by a set of matching templates. A black point is deletable if at least one template in the set of templates matches it. Templates are usually described by three kinds of elements, "black", "white", and "don't care", where "don't care" matches either black or white point in a given picture. In order to reduce the number of masks we use additional notations (see Fig. 4).

The first subiteration assigned to the deletion direction UD can delete certain $\mathbf{U}$ - or D-border points; the second subiteration associated with the deletion direction $\mathbf{N S}$ attempt to delete $\mathbf{N}$ - or $\mathbf{S}$-border points, and some $\mathbf{E}$ - or W-border points can be deleted by the third subiteration corresponding to the deletion direction $\mathbf{E W}$. The set of templates $\mathcal{T}_{\text {UD }}$ is given by Fig. 4 . Note that Fig. 4 shows only the eight base templates T1-T8. Additionally, all their rotations around the vertical axis belong to $\mathcal{T}_{\text {UD }}$, where the rotation angles are $90^{\circ}$, $180^{\circ}$, and $270^{\circ}$. This set of templates was constructed for deleting some simple points which are neither surface end-points (see Definition 1) nor extremities of surfaces. The deletable points of the other two subiterations (corresponding to deletion directions $\mathbf{N S}$ and $\mathbf{E W}$ ) can be obtained by proper rotations of the templates in $\mathcal{T}_{\text {UD }}$. Each template of our algorithm can be given by a Boolean condition that makes easy implementation possible.

Note that choosing another order of the deletion directions yields another algorithm. The proposed algorithm terminates when there are no more black points to be deleted. Since all considered input pictures are finite, it will terminate. 

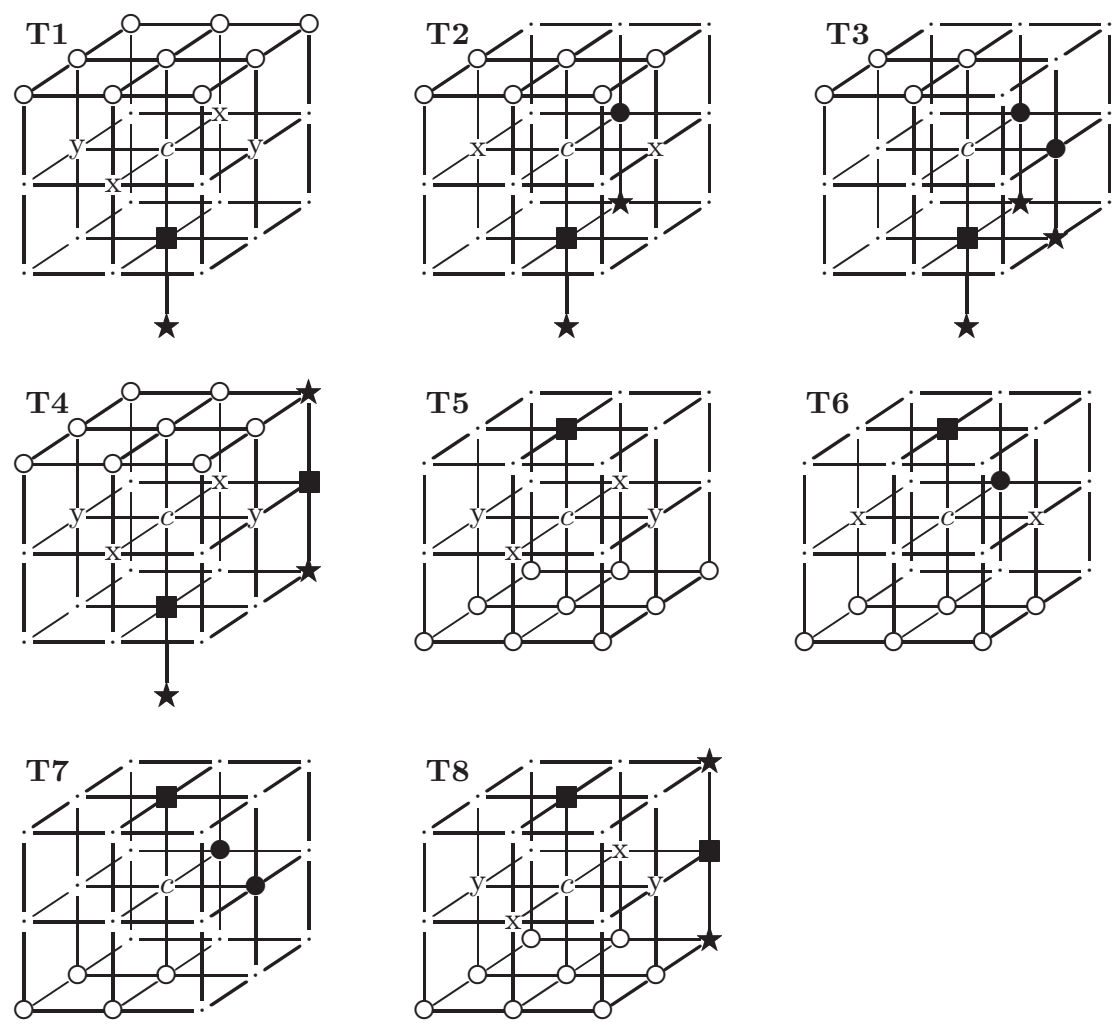

Fig. 4. Base templates $\mathbf{T} \mathbf{1}-\mathbf{T} 8$ and their rotations around the vertical axis form the set of templates $\mathcal{T}_{\text {UD }}$ assigned to the deletion direction UD. This set of templates belongs to the first subiteration. Notations: each position marked " $c$ ",

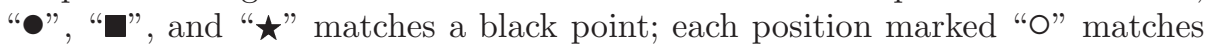
a white point; each "." ("don't care") matches either a black or a white point; at least one position marked " $\mathrm{x}$ " matches a black point; at least one position marked " $y$ " matches a black point. Emphasis is to be put that " $\mathbf{x}$ " and " $\mathbf{y}$ " positions provide that surface end-points cannot be deleted. (Note that using different symbols for black template positions helps us to prove the topological correctness of the algorithm)

\section{Discussion}

The proposed algorithm has been tested on objects of different shapes. Here we present only four examples (see Figs. 5-8).

The proposed 3-subiteration thinning algorithm is topology preserving for $(26,6)$ pictures. It is sufficient to prove that reduction operation given by the set 
of templates $\mathcal{T}_{\text {UD }}$ is topology preserving. If the first subiteration of the algorithm is topology preserving, then the other two are topology preserving, too, since the applied rotations of the deletion templates do not alter the topological properties. Therefore, the entire algorithm is topology preserving, since it is composed of topology preserving reductions.
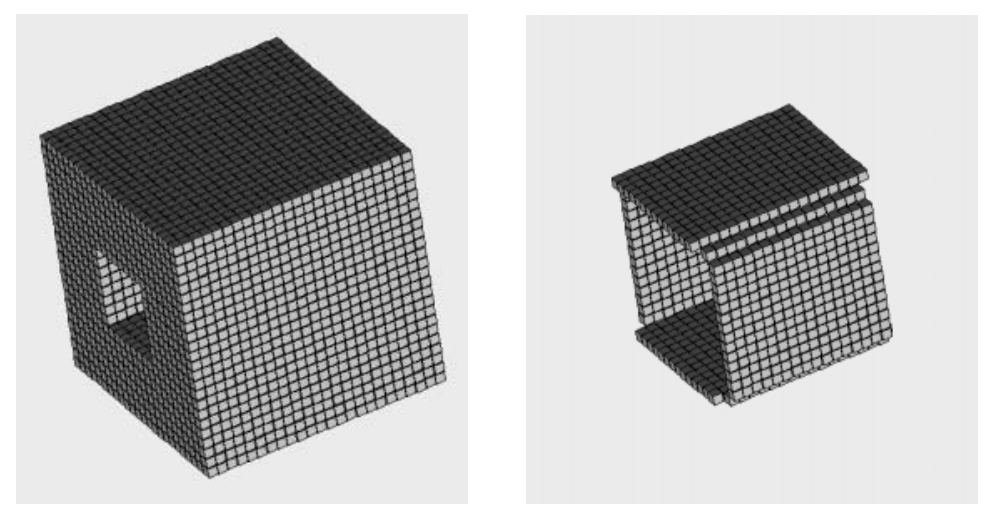

Fig. 5. A synthetic object containing a cube with a hole (left) and its medial surface produced by the proposed algorithm (right)
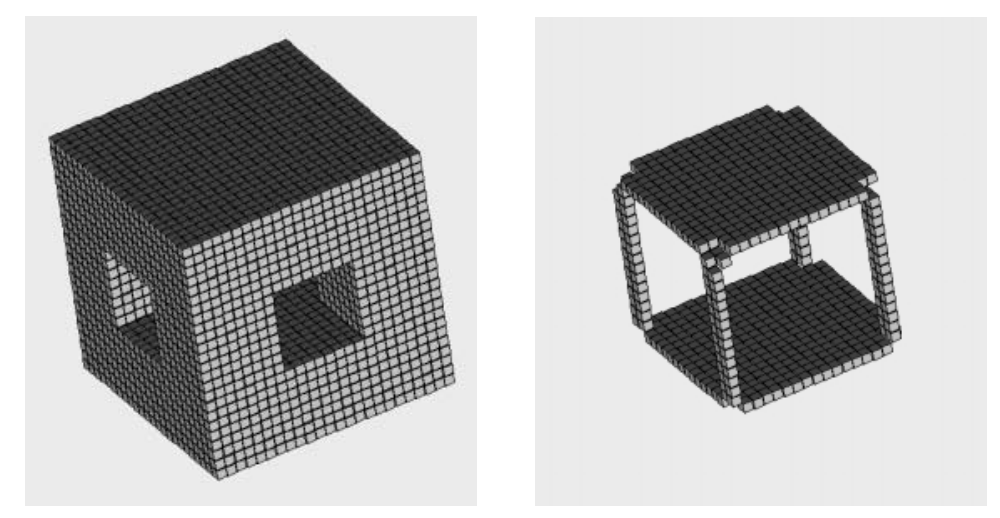

Fig. 6. A synthetic object containing a cube with two holes (left) and its medial surface produced by the proposed algorithm (right)

In order to prove both conditions of Theorem 2, we classify the elements of templates and state some properties of the set of templates $\mathcal{T}_{\text {UD }}$. The element in the very centre of a template is called central (marked by " $c$ " in Fig. 4). A 
noncentral template element is called black if it is always black (marked by

"ם", and " $\star$ " in Fig. 4). A noncentral template element is called white if it is always white (marked by "O" in Fig. 4). Any other noncentral template element which is neither white nor black, is called potentially black (marked by "x", "y", and "." in Fig. 4). A black or a potentially black noncentral template element is called nonwhite. A black point $p$ is deletable if it can be deleted by at least one template in $\mathcal{T}_{\mathrm{UD}} ; p$ is nondeletable otherwise.
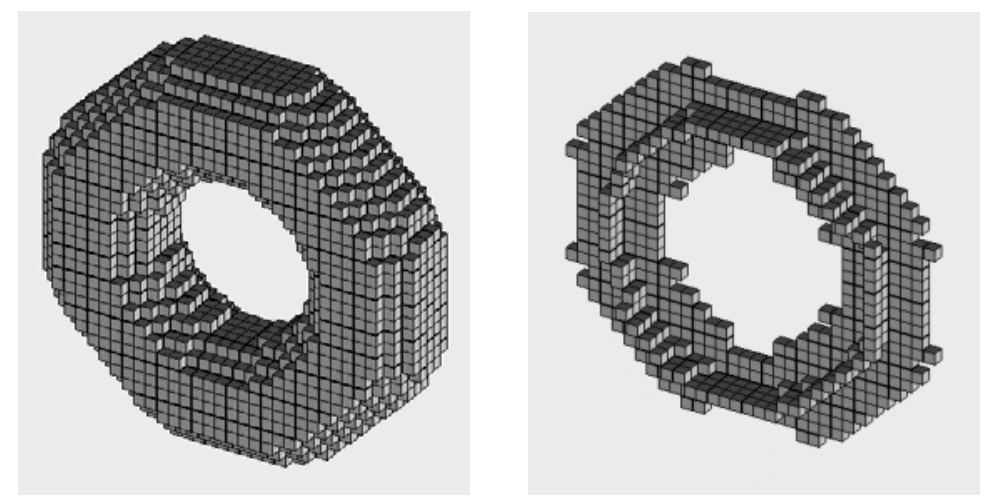

Fig. 7. A synthetic object containing a doughnut (left) and its medial surface produced by the proposed algorithm (right)

Observation 1. Let us examine the configurations illustrated in Fig. 9.

1. Black point $p$ in configuration (a) is nondeletable.

2. Black point $p$ in configuration (b) is deletable if

$-q=0, r=1$, and $s=1$, or

$-q=1$ and $r=0$.

3. Black point $p$ in configuration (c) is deletable if $q=1$ and $r=1$.

4. Black point $p$ in configuration (d) is deletable if $q=1$.

The topological correctness of the first subiteration of the proposed algorithm is stated by the following theorem:

Theorem 3. Reduction operation given by the set of templates $\mathcal{T}_{\mathrm{UD}}$ is topology preserving for $(26,6)$ pictures.

Proof. (sketch) It is easy to see that each template in $\mathcal{T}_{\text {UD }}$ deletes only simple points of $(26,6)$ pictures.

The first point is to verify that there exists a 26 -path between any two nonwhite positions (condition 1 of Theorem 1). It is sufficient to show that any 


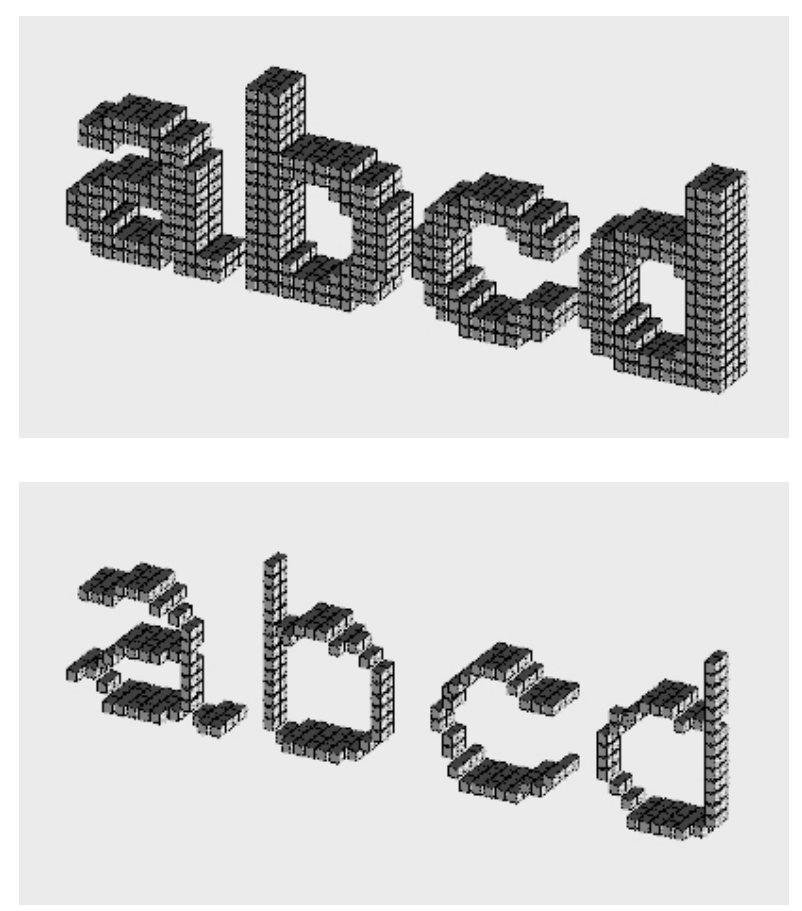

Fig. 8. A synthetic picture containing four characters (top) and their medial surface produced by the proposed algorithm (bottom)

potentially black position is 26 -adjacent to a black position and any black position is 26-adjacent to another black position. It is obvious by careful examination of the templates in $\mathcal{T}_{\text {UD }}$.

To prove that condition 2 of Theorem 1 holds, it is sufficient to show for each template in $\mathcal{T}_{\text {UD }}$ that:

1. there exists a white position 6 -adjacent to the central position,

2. for any two white positions 6-adjacent to the central position $p$ are 6 connected in the set of white positions 18-adjacent to $p$,

3. and for any potentially black position 6 -adjacent to the central position $p$, there exists a 6 -adjacent white 18 -neighbor which is 6-adjacent to a white position 6-adjacent to $p$.

The three points are obvious by a careful examination of the templates in $\mathcal{T}_{\text {UD }}$.

We know that each deletable point $p$ is simple. It can be stated that the value of any point coinciding with a potentially black template position does not alter the simplicity of $p$. We can state that the simplicity of a point $p$ does not depend on the points that coincide with a template position marked " $\star$ ", " $\mathrm{x}$ ", or "y" (see Fig. 4). In addition, black points that coincide with template positions 

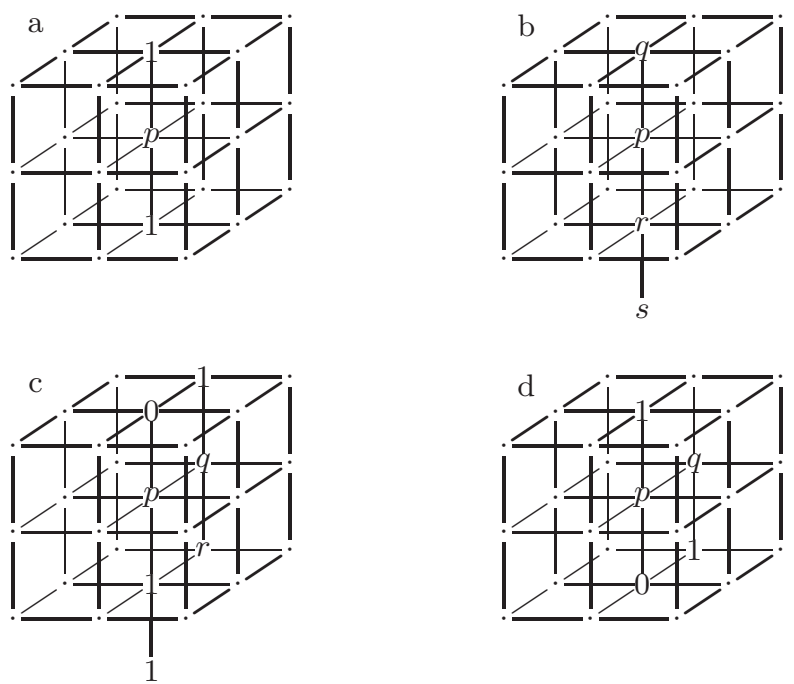

Fig. 9. Configurations assigned to Observation 1. Note that Observation 1 holds for their rotations around the vertical axis, too. (The rotation angles are $90^{\circ}$, $180^{\circ}$, and $270^{\circ}$.)

marked " $\square$ " are nondeletable (by Observation 1/1). Therefore, it is sufficient to deal with deletable points that coincide with template positions marked "•". Note that base templates T1, T4, T5, and T8 (and their rotated versions) do not contain any positions marked "•". Therefore, only base templates T2, T3, T6, and T7 (and their rotated versions) are to be investigated. It is easy to see with the help of Observation 1 that deletion of points coinciding with template positions marked "•" do not alter the simplicity of point $p$. Therefore, Condition 1 of Theorem 2 is satisfied.

Condition 2 of Theorem 2 can be seen with the help of Observation 1/2, too. Let us consider a unit lattice cube containing an upper set of four points $U=$ $\left\{u_{1}, u_{2}, u_{3}, u_{4}\right\}$ and a lower set of four points $L=\left\{l_{1}, l_{2}, l_{3}, l_{4}\right\}$. Let $C \subseteq U \cup L$ be a black component contained in the unit lattice cube. If $C \cap L$ contains a deletable point then $C \cap U \neq \emptyset$ by Observation $1 / 2$. It is easy to see, that any point in $C \cap U$ is nondeletable by Observation $1 / 2$. Therefore, black component $C$ cannot be deleted completely.

\section{Acknowledgements}

The author is grateful to Attila Kuba and László G. Nyúl for their valuable suggestions. This work was supported by OTKA T023804 and FKFP 0908/1997 Grants. 


\section{References}

1. Bertrand, G., Aktouf, Z.: A 3D thinning algorithms using subfields. In: Proc. SPIE Conference on Vision Geometry III, Vol. 2356 (1994) 113-124 410

2. Bertrand, G.: A parallel thinning algorithm for medial surfaces. Pattern Recognition Letters 16 (1995) 979-986 409, 410

3. Blum, H.: A transformation for extracting new descriptors of shape. Models for the Perception of Speech and Visual Form, MIT Press, (1967) 362-380 406

4. Gong, W. X., Bertrand, G.: A simple parallel 3D thinning algorithm. In: Proc. 10th International Conference on Pattern Recognition (1990) 188-190 409, 410

5. Kong, T. Y.: On topology preservation in $2-\mathrm{d}$ and $3-\mathrm{d}$ thinning. International Journal of Pattern Recognition and Artifical Intelligence 9 (1995) 813-844 408

6. Kong, T. Y., Rosenfeld, A.: Digital topology: Introduction and survey. Computer Vision, Graphics, and Image Processing 48 (1989) 357-393 406, 407, 408

7. Lee, T., Kashyap, R. L., Chu, C.: Building skeleton models via 3-D medial surface/axis thinning algorithms. CVGIP: Graphical Models and Image Processing 56 (1994) 462-478 409, 410

8. Ma, C. M.: On topology preservation in 3D thinning. CVGIP: Image Understanding 59 (1994) 328-339 408

9. Ma, C. M.: A 3D fully parallel thinning algorithm for generating medial faces. Pattern Recognition Letters 16 (1995) 83-87 409

10. Ma, C. M., Sonka, M.: A fully parallel 3D thinning algorithm and its applications. Computer Vision and Image Understanding 64 (1996) 420-433 409

11. Manzanera, A., Bernard, T. M., Pretêux, F., Longuet, B.: Medial faces from a concise 3D thinning algorithm. In: Proc. 7th IEEE Int. Conf. Computer Vision, ICCV'99 (1999) 337-343 409

12. Malandain, G., Bertrand, G.: Fast characterization of $3 \mathrm{D}$ simple points. In: Proc. 11th IEEE International Conference on Pattern Recognition (1992) 232-235 408

13. Morgenthaler, D. G.: Three-dimensional simple points: Serial erosion, parallel thinning and skeletonization. Technical Report TR-1005, Computer Vision Laboratory, Computer Science Center, University of Maryland (1981) 407

14. Mukherjee, J., Das, P. P., Chatterjee, B. N.: On connectivity issues of ESPTA. Pattern Recognition Letters 11 (1990) 643-648 409, 410

15. Palágyi K., Kuba, A.: A 3D 6-subiteration thinning algorithm for extracting medial lines. Pattern Recognition Letters 19 (1998) 613-627 409, 410

16. Palágyi K., Kuba, A.: A hybrid thinning algorithm for 3D medical images. Journal of Computing and Information Technology - CIT 6 (1998) 149-164 410

17. Palágyi K., Kuba, A.: Directional 3D thinning using 8 subiterations. In: Proc. 8th Int. Conf. on Discrete Geometry for Computer Imagery, DGCI'99, Lecture Notes in Computer Science, Vol. 1568. Springer (1999) 325-336 409

18. Palágyi K., Kuba, A.: A parallel 3D 12-subiteration thinning algorithm. Graphical Models and Image Processing 61 (1999) 199-221 409

19. Saha, P. K., Chaudhuri, B. B.: Detection of 3-D simple points for topology preserving transformations with application to thinning. IEEE Transactions on Pattern Analysis and Machine Intelligence 16 (1994) 1028-1032 408

20. Saha, P. K., Chaudhuri, B. B., Majumder, D. D.: A new shape-preserving parallel thinning algorithm for 3D digital images. Pattern Recognition 30 (1997) 1939-1955 410 
21. Székely, G.: Shape characterization by local symmetries. Habilitationsschrift, ETH Zürich (1996) 406

22. Tsao, Y. F., Fu, K. S.: A parallel thinning algorithm for 3-D pictures. Computer Graphics and Image Processing 17 (1981) 315-331 409, 410 